\title{
INATIVIDADE FÍSICA E COMPORTAMENTOS ADVERSOS PARA A SAÚDE ENTRE PROFESSORES UNIVERSITÁRIOS
}

\author{
PHYSICAL INACTIVITY AND ADVERSE HEALTH BEHAVIORS AMONG UNIVERSITY TEACHERS \\ LA INACTIVIDAD FISICA Y EL COMPORTAMIENTO ADVERSO PARA LA SALUD ENTRE LOS PROFESORES \\ UNIVERSITARIOS
}

Jaqueline de Oliveira Santana 1,2 (Profissional de Educação Física)

Sérgio Viana Peixoto 1,3

(Epidemiologista)

1. Centro de Pesquisas René Rachou - Fiocruz Minas (CPqRR), Belo Horizonte, MG, Brasil.

2. Instituto Federal de Ciência e Tecnologia de Minas Gerais (IFMG), Itabirito, MG, Brasil.

3. Universidade Federal de Minas Gerais (UFMG), Escola de Enfermagem, Belo Horizonte, MG, Brasil.

\section{Correspondência:}

Rua João Arantes, 152/201, Cidade Nova, Belo Horizonte, MG, Brasil. 31170-240.

jaquelineosantana@gmail.com

\begin{abstract}
RESUMO
Introdução: A inatividade física onera a sociedade pelo crescente custo dos cuidados médicos e perda de produtividade, além de figurar entre os quatro principais fatores de risco de doenças crônicas. Objetivo: Verificar os fatores associados à inatividade física, avaliada pela taxa de equivalentes metabólicos (MET), entre professores de uma universidade pública. Métodos: Estudo transversal, com amostra probabilística, incluindo 163 trabalhadores. Além da inatividade física (<450 MET.min/semana), avaliaram-se idade, sexo, estado civil, tabagismo, consumo excessivo de álcool, percepção da saúde e do estresse, presença de diabetes, hipertensão, lombalgia, depressão, doenças do coração e consumo de frutas/verduras/legumes, gordura não saudável, refrigerante e sal. As razões de prevalências e os intervalos de confiança (95\%) foram estimados pela regressão de Poisson. Resultados: Os participantes fisicamente inativos tinham menor consumo de frutas/verduras/legumes, consumo excessivo de álcool e pior percepção da saúde. Conclusão: Sugere-se que a inatividade física está associada a um maior perfil de risco de saúde, e a ocorrência simultânea desses fatores deve ser considerada no planejamento das ações de saúde para grupos de trabalhadores e população geral.
\end{abstract}

Descritores: atividade motora; exercício; fatores de risco; trabalhadores.

\section{ABSTRACT}

Introduction: Physical inactivity burdens society by increasing the costs of medical care and loss of productivity, and appears among the four major risk factors for chronic diseases. Objective: To identify the factors associated with physical inactivity, as assessed by metabolic equivalent rate (MET), among teachers of a public university. Methods: Cross-sectional study with probabilistic sample including 163 workers. In addition to physical inactivity ( $450 \mathrm{MET}$. min/week), we evaluated age, sex, marital status, smoking, excessive alcohol consumption, health and stress perception, diabetes, hypertension, low back pain, depression, heart disease and consumption of fruits/vegetables/legumes, unhealthy fat, soft drinks and salt. Prevalence ratios and confidence intervals (95\%) were estimated by Poisson regression. Results: The physically inactive participants had a lower intake of fruits/vegetables/legumes, higher alcohol consumption and worse perception of health. Conclusion: We suggest that physical inactivity is associated with a higher profile of health risk and that the simultaneous occurrence of these factors should be considered in the planning of health programs for groups of workers and general population.

Keywords: motor activity; exercise; risk factors; workers.

\section{RESUMEN}

Introducción: La inactividad física sobrecarga la sociedad debido al aumento de los costos de los cuidados médicos y la pérdida de productividad, además de figurar entre los cuatro principales factores de riesgo de enfermedades crónicas. Objetivo: Verificar los factores asociados a la inactividad física, evaluados por la tas a metabólica equivalente (MET) entre profesores de una universidad pública. Métodos: Estudio transversal, con una muestra probabilística, incluyendo 163 trabajadores. Además de la inactividad física (<450 MET.min/semana), se evaluó edad, sexo, estado civil, tabaquismo, consumo excesivo de alcohol, percepción de salud y el estrés, diabetes, hipertensión, dolor lumbar, depresión, enfermedad cardiaca y el consumo de frutas/verduras/legumbres, grasa no saludable, refrigerantes y sal. Las tasas de prevalencia e intervalos de confianza (95\%) se estimaron mediante regresión de Poisson. Resultados: Los participantes físicamente inactivos presentaron menor consumo de frutas/verduras/legumbres, mayor consumo de alcohol y peor percepción de la salud. Conclusión Se sugiere que la inactividad física se asocia con un perfil de mayor riesgo para la salud y que la ocurrencia simultánea de estos factores debe ser considerada en la planificación de acciones de salud para los grupos de trabajadores y la población en general.

Descriptores: actividad motora; ejercicio; factores de riesgo; trabajadores.

\section{INTRODUÇÃO}

Um total de 56 milhões de mortes ocorreram em todo o mundo durante o ano de 2012, sendo 38 milhões atribuídas a doenças crônicas não transmissíveis (DCNT). Os países de baixa e média renda são os 
mais afetados ${ }^{1}$, tornando-se um grande desafio para a saúde pública na atualidade 2 . No Brasil, cerca de $72 \%$ do total de óbitos foram atribuídos às DCNT, destacando-se as doenças cardiovasculares (DCV), as neoplasias, as doenças respiratórias e o diabetes ${ }^{3}$. Esse grupo de causas tem determinação complexa, mas os principais fatores de risco modificáveis são o tabagismo, a alimentação inadequada, o uso abusivo de bebidas alcoólicas e a inatividade física ${ }^{3,4}$, sendo que para os três últimos o país não apresenta uma evolução favorável nos últimos anos ${ }^{3,5}$.

A World Health Organization (WHO) define atividade física (AF) como sendo qualquer movimento corporal produzido pelos músculos esqueléticos que requeiram gasto de energia - incluindo atividades físicas praticadas durante o trabalho, jogos, execução de tarefas domésticas, viagens e em atividades de lazer. Por outro lado, a inatividade física (IF) é a ausência da prática de atividade física ou a prática insuficiente de atividade física (< 150 minutos por semana).

Estudos tem evidenciado que a atividade física regular tem potencial para melhorar a saúde, tendo efeitos positivos na prevenção das doenças cardiovasculares (DCV), alterando favoravelmente alguns fatores de risco, embora os resultados apresentem variações importantes entre diferentes populações ${ }^{6,7}$. Apesar de evidências dos benefícios da atividade física regular ${ }^{8}$, bem como dos efeitos deletérios da $\mathrm{IF}^{9}$, sua prevalência permanece alta em diversos países ${ }^{1}$. No Brasil, em 2013, 46,0\% dos adultos maiores de 18 anos eram insuficientemente ativos, ou seja, não cumpriam as recomendações de pelo menos 150 minutos de AF moderada a intensa por semana ${ }^{10}$.

Estudos desenvolvidos em diferentes populações mostram uma grande variação dos fatores associados à prática de atividade física, incluindo aspectos socioeconômicos, como idade, sexo, renda e escolaridade, e outros fatores de risco para DCNT, como tabagismo, consumo de álcool, dieta inadequada e piores condições de saúde $8,11-14$.

A inatividade física onera a sociedade através do crescente custo dos cuidados médicos e perda de produtividade ${ }^{15}$. Estudos epidemiológicos em diferentes estratos profissionais vêm apontando elevadas prevalências de fatores de risco para DCNT, mas a associação entre inatividade física e esses fatores de risco entre professores universitários ainda não foi explorada. Nesse grupo, os prejuízos econômicos e sociais advindos das DCNT podem ser maiores do que para a parcela de trabalhadores em geral, pois, espera-se que estes profissionais atinjam seu ápice profissional justamente após os 40 anos de idade, período em que as DCNT têm maior prevalência e podem se manifestar de forma aguda provocando incapacidade física ou morte ${ }^{16}$

Nesse contexto, este estudo teve como objetivo analisar os fatores associados à inatividade física em uma população de professores universitários.

\section{MATERIAIS E MÉTODOS}

Estudo transversal entre professores do ensino superior com vínculo empregatício (professores efetivos) na Universidade Federal de Ouro Preto (UFOP), que dispõe de unidades acadêmicas em três campi, localizados nas cidades de Ouro Preto, Mariana e João Monlevade (campus avançado). O maior campus é localizado no município de Ouro Preto (MG), cidade com uma área de 1.245,86 km², declarada "Patrimônio da Humanidade" pela Organização das Nações Unidas para a Educação, a Ciência e a Cultura (UNESCO), e abriga uma população de aproximadamente 74.036 mil habitantes ${ }^{17}$. Em 2013, a UFOP apresentava 42 cursos de graduação (29 em Ouro Preto; nove em Mariana; quatro em João Monlevade), 41 cursos de pós-graduação stricto sensu (35 em Ouro Preto e seis em Mariana) e 17 cursos de pós-graduação lato sensu, contemplando as diferentes áreas do conhecimento (ciências exatas, humanidades e biomédicas). Considerando os três campi, mais de 15 mil alunos estavam matriculados, cerca de 800 técnicos-administrativos e 800 professores estavam vinculados à UFOP. Nesse período, 595 professores efetivos lotavam o campus Ouro Preto.

A amostra foi calculada com base na prevalência de IF de 30\%", erro amostral de 5\% e intervalo de confiança de 95\% (IC95\%). Houve acréscimo de 20\% para perdas e recusas, totalizando 232 professores. Posteriormente, foi feito sorteio aleatório simples desses trabalhadores, utilizando-se uma lista fornecida pela UFOP. Da amostra inicialmente sorteada, participaram desse estudo 163 professores universitários.

O projeto foi aprovado pelo Comitê de ética em pesquisa da UFOP e da Universidade Federal de Minas Gerais-UFMG (CAAE: 08604212.5.3001.5150) e todos os participantes assinaram o termo de consentimento livre e esclarecido.

As informações avaliadas nesse estudo foram obtidas por meio de entrevista (entre setembro de 2013 e fevereiro de 2015), previamente agendada com cada professor, utilizando-se questionário padronizado e entrevistadores treinados, sendo conduzida no local de trabalho do professor.

O questionário incluiu informações demográficas (idade, sexo, estado civil), comportamentos em saúde (tabagismo atual, consumo abusivo de álcool, hábitos alimentares) e condições de saúde (percepção da saúde, do estresse, e doenças autorreferidas), baseando-se no questionário da pesquisa VIGITEL (2010), exceto as questões referentes à prática de atividade física e à percepção de estresse.

Para o hábito de fumar, foram considerados fumantes atuais os participantes que relataram ser fumante no momento da entrevista, independente da quantidade e frequência ("O sr (a) fuma atualmente?"). O consumo abusivo de bebidas alcoólicas foi avaliado pela seguinte pergunta: "Nos últimos 30 dias, o sr. chegou a consumir 5 ou mais doses de bebida alcoólica em uma única ocasião ( 5 doses de bebida alcoólica seriam 5 latas de cerveja, 5 taças de vinho ou 5 doses de cachaça, whisky ou qualquer outra bebida destilada)?"; para mulheres, considerou-se quatro ou mais doses.

Os hábitos alimentares foram avaliados pela frequência semanal do consumo de frutas ("Em quantos dias da semana o (a) sr (a) costuma comer frutas?"), verduras e legumes ("Em quantos dias da semana o (a) sr (a) costuma comer pelo menos um tipo de verdura ou legume - alface, tomate, couve, cenoura, chuchu, berinjela, abobrinha - não vale batata, mandioca ou inhame?") e refrigerante ("Em quantos dias da semana o(a) sr(a) costuma tomar refrigerante ou suco artificial?"), considerando-se essas variáveis categorizadas em cinco ou mais dias por semana e consumo de menor frequência. Também foi considerado se o participante consumia carnes (vermelha e frango) com gordura visível ("Quando o (a) sr (a) come carne vermelha o (a) sr (a)" e "Quando o (a) sr (a) come frango/ galinha o (a) sr (a)" - tira a gordura/pele, come com a gordura/pele ou não come) e leite com teor integral de gorduras ("Quando o sr (a) toma leite, que tipo de leite costuma tomar?"), além de adição de sal à comida ("O sr (a) costuma colocar mais sal nos alimentos quando já servidos em seu prato?"), sendo essas variáveis categorizadas como sim e não.

Os entrevistados foram questionados quanto à presença das seguintes condições crônicas, diagnosticadas por um médico ("Algum médico já lhe disse que o sr (a) tem:"): hipertensão arterial, diabetes, dislipidemia, lombalgia, depressão e doenças do coração. A percepção da saúde foi avaliada pelo auto relato sobre o próprio estado de saúde ("Como você avalia a sua saúde atualmente?") e foi categorizada em boa/muito boa e regular/ ruim/muito ruim. A auto percepção de estresse foi também considerada ("O sr (a) se sente estressado com qual frequência?"), considerando-se as categorias nunca/raramente/às vezes e quase sempre/sempre.

Para avaliar o nível de AF foi utilizado o questionário IPAQ (International Physical Activity Questionnaire) em sua versão reduzida, traduzida e validada para o Brasil ${ }^{18}$. O instrumento contém perguntas relacionadas à frequência (dias por semana), duração (tempo por dia) e intensidade 
(leve, moderada e vigorosa) da AF nos últimos sete dias. As informações coletadas pelo IPAQ foram convertidas em taxa de equivalentes metabólicos (METs), baseando-se no Compêndio de AF ${ }^{19}$. A inatividade física (variável dependente) foi caracterizada por um gasto energético em atividades físicas inferior a 450 MET. min/semana ${ }^{20}$.

\section{Análise estatística}

Foi realizada uma análise descritiva das variáveis consideradas nesse estudo, utilizando percentual e média (desvio padrão), além da verificação da associação de cada uma dessas variáveis com a inatividade física, utilizando o teste do Qui-quadrado para comparação de proporções ou o teste $t$ de Student para comparação de médias.

Para a análise ajustada foi utilizada a regressão de Poisson, com variância robusta, seguindo um modelo hierárquico com ajustes progressivos, estimando-se razões de prevalência e IC 95\%. Foram testados três modelos: a) modelo ajustado por todos os fatores demográficos; b) modelo com os comportamentos em saúde, ajustado pelos fatores demográficos; e c) modelo com as condições de saúde, ajustado pelas variáveis dos dois blocos anteriores. A variável diabetes foi excluída dessa análise, pois o pequeno número de professores com essa condição não possibilitou a estimativa da força de associação. Para todas as análises, foi adotado o nível de significância de 5\%.

Os dados foram digitados e conferidos no EpiData e as demais análises foram realizadas no Programa Stata 13.0.

\section{RESULTADOS}

Entre os 163 participantes do estudo, 107 (65,6\%) eram homens e 56 (34,4\%) mulheres. A média de idade foi igual a 43,9 anos (desvio padrão $=9,9$ anos). A população estudada apresentou um gasto energético médio igual a 1565,2 MET.min/semana, sendo 30,7\% (IC95\%: 24,0\% - 38,3\%) classificados como sedentários.

A Tabela 1 descreve as características demográficas e comportamentais da população estudada. A maioria dos professores era casada $(75,5 \%)$, não fumante $(92,6 \%)$ e não faziam uso excessivo de álcool (66,9\%). Nesse grupo de trabalhadores, 95,1\% relataram consumo de frutas/verduras/legumes em pelo menos cinco dias na semana e 58,9\% relataram consumo de carne/frango com gordura visível e/ou leite com teor integral de gordura. A maioria não adicionava sal à comida (86,5\%) e consumiam refrigerante menos de cinco vezes na semana $(89,0 \%)$. Associações significativas com inatividade física foram observadas para consumo excessivo de álcool $(p=0,007)$ e consumo de frutas, verduras e legumes $(p=0,045)$.

A Tabela 2 mostra as variáveis relacionadas à condição de saúde segundo a inatividade física. Entre os participantes, 17,4\% relataram hipertensão arterial, 2,5\% diabetes, 30,7\% dislipidemia, 33,3\% lombalgia, $6,2 \%$ depressão e 3,3\% doenças do coração. Referiram uma pior percepção de estresse 29,4\%, e uma pior percepção da saúde 24,5\% dos trabalhadores. Associação significativa foi observada entre inatividade física e pior percepção da saúde ( $p<0,001)$.

O modelo de regressão múltipla da associação entre o sedentarismo e características demográficas é apresentado na Tabela 3, não sendo observadas associações significativas, de forma semelhante ao observado na análise univariada.

A Tabela 4 mostra os resultados do modelo de regressão múltipla da associação entre inatividade física e os comportamentos em saúde. A prevalência desse evento foi maior entre aqueles que reportaram consumo excessivo de álcool (RP: 1,82; IC95\%: 1,12-2,96) e entre os que relataram menor consumo de frutas, verduras ou legumes (RP: 2,20; IC95\%: 1,23-3,94).

A Tabela 5 apresenta os resultados da associação entre a inatividade física e a saúde autorreferida, considerando-se o ajuste por todas as variáveis incluídas no estudo. Nesse modelo, observou-se maior
Tabela 1. Características demográficas e comportamentais entre professores universitários. Ouro Preto, Minas Gerais, 2015.

\begin{tabular}{c|c|c|c|c|c}
\hline Variáveis & \multirow{2}{*}{ Total } & \multicolumn{2}{|c|}{ Inatividade física } & Valor do & \multirow{2}{*}{$\begin{array}{c}\text { Valor } \\
\text { teste* }\end{array}$} \\
\cline { 3 - 4 } $\mathbf{p}^{*}$
\end{tabular}

Valores expressos em número (\%), exceto quando especificado. * Teste do Qui-quadrado de Pearson ou teste de Student.

Tabela 2. Condições de saúde autoreferidas entre professores universitários. Ouro Preto, Minas Gerais, 2015

\begin{tabular}{|c|c|c|c|c|c|}
\hline \multirow[t]{2}{*}{ Variáveis } & \multirow{2}{*}{$\begin{array}{l}\text { Total } \\
\mathrm{N}(\%) \\
\end{array}$} & \multicolumn{2}{|c|}{$\begin{array}{c}\text { Inatividade } \\
\text { física - N (\%) }\end{array}$} & \multirow{2}{*}{$\begin{array}{c}\text { Valor do } \\
\text { teste* }\end{array}$} & \multirow{2}{*}{$\begin{array}{c}\text { Valor } \\
\text { p* }^{*}\end{array}$} \\
\hline & & Sim & Não & & \\
\hline \multicolumn{6}{|l|}{ Hipertensão arterial } \\
\hline $\operatorname{Sim}$ & $28(17,4)$ & $8(16,0)$ & $20(18,0)$ & \multirow{2}{*}{0,10} & \multirow{2}{*}{0,755} \\
\hline Não & $133(82,6)$ & $42(84,0)$ & $91(82,0)$ & & \\
\hline \multicolumn{6}{|l|}{ Diabetes } \\
\hline Sim & $4(2,5)$ & $0(0,0)$ & $4(3,5)$ & \multirow{2}{*}{1,74} & \multirow{2}{*}{0,187} \\
\hline Não & $157(97,5)$ & $48(100,0)$ & $109(96,5)$ & & \\
\hline \multicolumn{6}{|l|}{ Dislipidemia } \\
\hline $\operatorname{Sim}$ & $47(30,7)$ & $18(40,9)$ & $29(26,6)$ & \multirow{2}{*}{3,01} & \multirow{2}{*}{0,083} \\
\hline Não & $106(69,3)$ & $26(59,1)$ & $80(73,4)$ & & \\
\hline \multicolumn{6}{|l|}{ Lombalgia } \\
\hline Sim & $54(33,3)$ & $17(34,0)$ & $37(33,0)$ & \multirow{2}{*}{0,01} & \multirow{2}{*}{0,904} \\
\hline Não & $108(66,7)$ & $33(66,0)$ & $75(67,0)$ & & \\
\hline \multicolumn{6}{|l|}{ Depressão } \\
\hline Sim & $10(6,2)$ & $3(6,2)$ & $7(6,2)$ & \multirow{2}{*}{0,01} & \multirow{2}{*}{0,989} \\
\hline Não & $151(93,8)$ & $45(93,8)$ & $106(93,8)$ & & \\
\hline \multicolumn{6}{|l|}{ Doenças do coração } \\
\hline $\operatorname{Sim}$ & $5(3,3)$ & $2(4,4)$ & $3(2,8)$ & \multirow{2}{*}{0,26} & \multirow{2}{*}{0,612} \\
\hline Não & $146(96,7)$ & $43(95,6)$ & $103(97,2)$ & & \\
\hline \multicolumn{6}{|l|}{ Percepção da saúde } \\
\hline Bom/muito bom & $123(75,5)$ & $28(56,0)$ & $95(84,1)$ & \multirow{2}{*}{14,75} & \multirow{2}{*}{$\begin{array}{c}< \\
0,001 \\
\end{array}$} \\
\hline Regular/ruim/muito ruim & $40(24,5)$ & $22(44,0)$ & $18(15,9)$ & & \\
\hline \multicolumn{6}{|l|}{ Estresse } \\
\hline Raro/nunca/às vezes & $115(70,6)$ & $32(64,0)$ & $83(73,4)$ & \multirow{2}{*}{1,49} & \multirow[t]{2}{*}{0,222} \\
\hline Sempre/quase sempre & $48(29,4)$ & $18(36,0)$ & $30(26,6)$ & & \\
\hline
\end{tabular}


prevalência de inatividade física entre aqueles que relataram uma pior percepção da própria saúde (RP: 2,29; IC95\%: 1,32-4,00).

\section{DISCUSSÃO}

A prevalência de inatividade física entre professores universitários foi de 30,7\%, estando dentro da variação observada em estudos recentes entre trabalhadores brasileiros da área da saúde, nos quais essa prevalência parece ser menor do que em outros estratos profissionais, variando entre $14,1 \%$ e $35,4 \%{ }^{2,12}$. Valores superiores foram observados na Pesquisa Nacional de Saúde (PNS) 21 e no Vigitel22 em 2013, nas quais a proporção de adultos insuficientemente ativos foi de $46,0 \%$ e $49,6 \%$, respectivamente. Outros estudos realizados entre trabalhadores ${ }^{23,24}$, tem apontado prevalências elevadas de sedentarismo, variando entre $45,6 \%$ e $76,5 \%$.

O consumo excessivo de álcool $(33,1 \%)$ entre os professores foi superior ao observado entre adultos da população geral $(16,4 \%)$ no ano de $2013^{22}$, mas ressalta-se que diferentes critérios são utilizados para caracterizar consumo excessivo de bebidas alcoólicas ${ }^{12}$. Poucos são os estudos que investigaram a relação entre sedentarismo e consumo abusivo de álcool, sendo os resultados contraditórios ${ }^{12}$. Enquanto um estudo entre trabalhadores mostra associação significativa e negativa entre a prática de AF e o consumo de álcool ${ }^{12}$, concordando com os resultados do presente estudo, outro não observou essa associação ${ }^{25}$. De forma oposta ao observado no presente trabalho, estudos de revisão recentes mostram associações positivas entre a prática de AF e o consumo de álcool moderado ou excessivo ${ }^{13}$. A associação entre AF e consumo excessivo de álcool pode parecer paradoxal, porém, estudos utilizando metodologias diferentes para mensurar esse consumo tem

Tabela 3. Associação entre as características demográficas e a inatividade física entre professores universitários. Ouro Preto, MG, 2015.

\begin{tabular}{c|c}
\hline Variáveis & RP (IC 95\%) \\
\hline Idade & $0,99(0,97-1,02)$ \\
\hline Sexo & 1,00 \\
\hline Feminino & $1,33(0,78-2,27)$ \\
\hline Masculino & 1,00 \\
\hline Estado civil & $1,11(0,62-1,98)$ \\
\hline Não casado & \\
\hline Casado &
\end{tabular}

RP (95\% Cl): Razões de prevalência e intervalos de confiança de 95\% estimados pela regressão de Poisson, ajustada pelas variáveis listadas na tabela.

Tabela 4. Associação entre os comportamentos em saúde e a inatividade física entre professores universitários. Ouro Preto, MG, 2015.

\begin{tabular}{|c|c|}
\hline Variáveis & RP (IC 95\%) \\
\hline \multicolumn{2}{|l|}{ Tabagismo atual } \\
\hline Sim & $0,90(0,42-1,94)$ \\
\hline Não & 1,00 \\
\hline \multicolumn{2}{|c|}{ Consumo excessivo de álcool } \\
\hline Sim & $1,82(1,12-2,96)^{*}$ \\
\hline Não & 1,00 \\
\hline \multicolumn{2}{|l|}{$\begin{array}{l}\text { Consumo de frutas, } \\
\text { verduras ou legumes }\end{array}$} \\
\hline$<5$ dias na semana & $2,20(1,23-3,94)^{*}$ \\
\hline$\geq 5$ dias na semana & 1,00 \\
\hline \multicolumn{2}{|c|}{$\begin{array}{l}\text { Consumo de carne/frango com } \\
\text { gordura visível e/ou leite integral }\end{array}$} \\
\hline $\operatorname{Sim}$ & $0,81(0,51-1,29)$ \\
\hline Não & 1,00 \\
\hline \multicolumn{2}{|l|}{ Adição de sal à comida } \\
\hline $\operatorname{Sim}$ & $0,77(0,39-1,53)$ \\
\hline Não & 1,00 \\
\hline \multicolumn{2}{|l|}{ Consumo de refrigerante } \\
\hline$\geq 5$ dias na semana & $1,65(0,88-3,13)$ \\
\hline$<5$ dias na semana & 1,00 \\
\hline
\end{tabular}

RP (95\% Cl): Razões de prevalência e intervalos de confiança de 95\% estimados pela regressão de Poisson, ajustada pelos fatores demográficos e demais variáveis listadas na tabela. * $p<0,05$. suportado essa evidência ${ }^{13}$. Essa associação pode derivar em parte do fato de que ambos representam estímulos recompensadores que atuam na via mesocorticolímbica do cérebro, sendo o exercício uma recompensa natural, ao contrário do álcool. Isto sugere que o exercício pode ser de utilidade limitada como um método de intervenção no tratamento de indivíduos com dependência de álcool ${ }^{13}$.

Apesar das discordâncias entre os estudos, entre as metas da OMS para a prevenção de DCNT no período de 2013 a 2020, está a redução do consumo médio anual de álcool em 10\%, considerando sua associação com diversos agravos à saúde. Portanto, a relação entre álcool, AF e saúde é complexa e multifacetada, e até que se tenha uma melhor compreensão desse aspecto, em termos de prevenção primária de DCNT, permanecem as recomendações da WHO que envolve a diminuição do uso de álcool e do sedentarismo.

Em relação aos hábitos alimentares, evidências indicam que uma dieta de baixa qualidade, com baixo consumo de frutas e verduras e alto de gordura saturada, são importantes fatores de risco para DCNT26 e mortalidade em todo o mundo. No presente estudo, 95,1\% relataram o consumo de frutas, verduras ou legumes em cinco dias ou mais na semana. De forma contrária, estudos mostram uma baixa prevalência de consumo de frutas e verduras (menos de cinco dias na semana) entre profissionais da indústria $(44,0 \%)^{23}$ e de verduras entre profissionais da área da saúde $(18,4 \%)^{2}$, mas comparações mais detalhadas entre os estudos são dificultadas pelo uso de metodologias diferentes 11,21,22,23. Apesar disso, baixo consumo de frutas e verduras foi observado tanto na população geral ${ }^{11,22}$ quanto entre trabalhadores ${ }^{11}$.

Florindo et al. ${ }^{11}$ mostraram uma associação direta entre nível de AF e consumo de frutas e verduras entre adultos' enquanto Pinho et al. ${ }^{27}$ não observaram essa associação'. Na mesma direção do que foi observado entre trabalhadores da área da saúde ${ }^{11}$, na presente investigação foi observada associação significativa entre inatividade física e menor consumo de frutas e verduras. Esse fato corrobora a possível existência concomitante de comportamentos que favorecem a ocorrência de DCNT ${ }^{28}$, nesse caso, uma dieta pobre em frutas, verduras e legumes e a inatividade física.

O percentual de pior percepção da própria saúde na população de professores universitários (24,5\%) foi semelhante ao observado na

Tabela 5. Associação entre as condições de saúde autoreferidas e a inatividade física entre professores universitários. Ouro Preto, MG, 2015.

\begin{tabular}{|c|c|}
\hline Variáveis & RP (IC 95\%) \\
\hline \multicolumn{2}{|l|}{ Hipertensão arterial } \\
\hline Sim & $0,68(0,31-1,48)$ \\
\hline Não & 1,00 \\
\hline \multicolumn{2}{|l|}{ Dislipidemia } \\
\hline Sim & $1,36(0,77-2,43)$ \\
\hline Não & 1,00 \\
\hline \multicolumn{2}{|l|}{ Lombalgia } \\
\hline Sim & $1,00(0,56-1,78)$ \\
\hline Não & 1,00 \\
\hline \multicolumn{2}{|l|}{ Depressão } \\
\hline Sim & $0,73(0,26-2,01)$ \\
\hline Não & 1,00 \\
\hline \multicolumn{2}{|l|}{ Doenças do coração } \\
\hline Sim & $1,11(0,41-3,02)$ \\
\hline Não & 1,00 \\
\hline \multicolumn{2}{|l|}{ Percepção da saúde } \\
\hline Regular/ruim/muito ruim & $2,29(1,32-4,00)^{*}$ \\
\hline Boa/Muito boa & 1,00 \\
\hline \multicolumn{2}{|l|}{ Percepção de estresse } \\
\hline Quase sempre/sempre & $0,84(0,47-1,51)$ \\
\hline Raramente/nunca/às vezes & 1,00 \\
\hline
\end{tabular}

RP (95\% Cl): Razões de prevalência e intervalos de confiança de 95\% estimados pela regressão de Poisson, ajustada pelos fatores demográficos, comportamentos em saúde e demais variáveis listadas na tabela. ${ }^{*} p<0,05$. 
população de adultos da região Sudeste (28,2\%) em $2013^{21}$ e maior que adultos dos Estados Unidos (12,0\%) em 201229. Um relato de uma pior percepção da saúde entre adultos tende a diminuir com o aumento da escolaridade ${ }^{14,21,22,29}$, o que pode explicar os resultados do presente estudo. A associação da autoavaliação negativa de saúde com inatividade física observada entre os professores participantes desse estudo corrobora achado recente de outra investigação entre adultos da população geral ${ }^{14}$ e entre trabalhadores ${ }^{8}$. No entanto, nos estudos entre trabalhadores, foi observada associação entre o sedentarismo no lazer e a percepção da saúde, e não em todos os domínios, como no presente estudo. Como a autoavaliação é uma medida global da saúde, incluindo não apenas aspectos físicos, mas também no nível de bem-estar geral ${ }^{14}$, essa associação reforça os benefícios da prática da AF regular, favorecendo não apenas a prevenção e controle de DCNT, mas também melhorando o estado geral de saúde, incluindo seus aspectos psicológicos e sociais ${ }^{14}$.

Algumas limitações desse estudo devem ser consideradas. O desenho seccional não permite estabelecer uma relação temporal entre as variáveis pesquisadas. A utilização do auto relato para avaliar o gasto energético em atividades físicas pode gerar erro nessa estimativa, embora tenha sido utilizado um questionário padronizado e validado para o Brasil. A homogeneidade da amostra (situação socioeconômica e escolaridade semelhantes), pode gerar similaridade em relação a esses fatores e também às outras variáveis exploratórias consideradas na análise, dificultando a observação de associações significativas. Além disso, deve-se considerar a perda amostral (29,7\%), devido à dificuldade de contato com os professores e à negativa de participação na pesquisa. Por outro lado, este é um estudo que considerou uma população específica de trabalhadores, e que incluiu as atividades físicas realizadas em todos os domínios, e não apenas no lazer, utilizando-se um questionário validado. Explorou-se um conjunto de fatores que podem ter um impacto no futuro da saúde de adultos economicamente ativos, considerando a escassez de estudos em populações específicas. A utilização de uma amostra probabilística e não de conveniência, como em muitos outros estudos entre trabalhadores é uma vantagem adicional.

Nos últimos anos, o Brasil tem empreendido diversos esforços para o enfrentamento de DCNT, tendo pautado o aumento da prática de AF como um dos pontos-chave desse processo. Algumas ações merecem destaque, como a Política Nacional de Promoção da Saúde e o Plano de Ações Estratégicas para o Enfrentamento das DCNT no Brasil ${ }^{4}$. A prática de AF tem recebido atenção especial nesses documentos, com diretrizes específicas voltadas ao aumento dos níveis de AF na população geral. Porém, poucas são as ações que buscam estimular a prática de AF voltadas para o cuidado à saúde do trabalhador, que poderiam resultar em benefícios diretos na prevenção de DCNT, aumento da produtividade e diminuição do absenteísmo.

Identificar os grupos populacionais mais expostos a fatores e comportamentos de risco, como o sedentarismo, é uma das informações necessárias para elaborar ações que objetivem reduzi-los em nível populacional. Sabe-se que estratégias de incentivo à prática de AF que desconsideram as peculiaridades dos subgrupos, uniformizando de forma demasiada suas metas e ações, não são eficazes ${ }^{30}$.

\section{CONCLUSÕES}

Em geral, os resultados do presente estudo sugerem que a inatividade física está associada a um maior perfil de risco para DCNT, o que fala a favor do incentivo à prática de AF e de estratégias que promovam de forma efetiva mudanças no estilo de vida da população, sobretudo no âmbito da saúde do trabalhador. Nesse contexto, os resultados poderão contribuir para o planejamento de ações mais bem direcionadas e mais eficientes para o gerenciamento de prioridades necessárias à saúde do indivíduo, tanto para a organização que o emprega, como para si mesmo e a sociedade como um todo.

\section{AGRADECIMENTOS}

À Fundação de Amparo à Pesquisa do Estado de Minas Gerais pelo apoio financeiro (FAPEMIG CDS-APQ- 01899-12). À Kerllyn T. Coimbra, Ketlen T. de Faria, Marcone Rodrigues, Ana Daniela Damacena, Nayara Figueiredo, Taís Carvalho, Eliana J. de Paula e Gustavo Eleutério pela colaboração na coleta de dados para a pesquisa. SVP é bolsista de produtividade do Conselho Nacional de Desenvolvimento Científico e Tecnológico (CNPq).

Todos os autores declararam não haver qualquer potencial conflito de interesses referente a este artigo.

CONTRIBUIÇÕES DE AUTORES: Cada autor contribuiu individual e significativamente para o desenvolvimento do manuscrito. JOS (0000-0002-4741-2390)* participou da concepção do projeto, coleta, análise, interpretação, e discussão dos dados, redação e revisão crítica do conteúdo do manuscrito. SVP (0000-0001-9431-2280** participou da concepção do projeto, análise e discussão dos dados, redação e revisão crítica do conteúdo do manuscrito, revisão e aprovação final da versão do manuscrito. *ORCID (Open Researcher and Contributor ID).

\section{REFERÊNCIAS}

1. World Health Organization. Global health observatory data repository, 2012 [acesso em 2016 fev 05]. Disponivel em: http://apps.who.int/gho/data/node.main.A867?lang=en.

2. Chaves CS, Leitão MPC, Braga Junior ACR, Sirino ACA. Identificação defatores de risco para doenças cardiovasculares em profissionais da saúde. Arq Ciênc Saúde. 2015; 22(1):39-47.

3. Schmidt MI, Duncan BB, Azevedo e Silva G, Menezes AM, Monteiro CA, Barreto SM, et al. Chronic non-communicable diseases in Brazil: burden andcurrent challenges. Lancet. 2011;377(9781):1949-61.

4. Ministério da Saúde, Secretaria de Vigilância em Saúde. Plano de ações estratégicas para o enfrentamento das doenças crônicas não transmissíveis (DCNT) no Brasil 2011-2022. Ministério da Saúde; 2011 [acesso em 2016 set 26]. Disponível emhttp://portal.saude.gov.br/portal/arquivos/pdf/cartilha_dcnt_pequena_portugues_espanhol.pdf.

5. Duncan BB, Chor D, Aquino EM, Bensenor IM, Mill JG, Schmidt MI, et al. Chronic non-communicable diseases in Brazil: priorities for disease management and research. Rev Saude Publica. 2012;46(Suppl 1):126-34.

6. Wen CP, Wai JP, Tsai MK, Yang YC, Cheng TY, Lee MC, et al. Minimum amount of physical activity for reduced mortality and extended life expectancy: a prospective cohort study. Lancet. 2011;378(9798):1244-53.

7. Durstine JL, Gordon B, Wang Z, Luo X. Chronic disease and the link tophysical activity. J Sport Health Sci. 2013;2(1)3-11.

8. Bogaert I, De Martelaer K, Deforche B, Clarys P, Zinzen E. Associations between different types of physical activity and teachers' perceived mental, physical, and work-related health. BMC Public Health. 2014;14:534.

9. de Rezende LF, Rabacow FM, Viscondi JY, Luiz Odo C, Matsudo VK, Lee IM. Effectof physical inactivity on major noncommunicable diseases and life expectancy inBrazil. J Phys Act Health. 2015;12(3):299-306.

10. Mielke GI, Hallal PC, Rodrigues GBA, Szwarcwald CL, Santos FV, Malta DC. Prática de atividade física e hábito de assistir à televisão entre adultos no Brasil: Pesquisa Nacional de Saúde 2013. Epidemiol Serv Saúde.2015;24(2):277-86.
11. Florindo AA, Brownson RC, Mielke Gl, Gomes GA, Parra DC, Siqueira FV, et al. Association of knowledge, preventive counseling and personal health behaviors on physical activity andconsumption of fruits or vegetables in community health workers. BMC Public Health. 2015;15:344.

12. Codarin MAF, Moulatlet EM, NehmeP, Ulhôa M, Moreno CRC. Associação entre prática de atividade física, escolaridade e perfil alimentar de motoristas decaminhão. Saude Soc. 2010;19(2):418-28.

13. Leasure JL, Neighbors C, Henderson CE, Young CM. Exercise and alcohol consumption: what we know, what we need to know, and why it is important. FrontPsychiatry. 2015;6:156.

14. Bezerra PC, Opitz SP, Koifman RJ, Muniz PT. Self-rated health and associated factors in adults: a population survey in Rio Branco, Acre State, Brazil, 2007-2008. Cad Saude Publica. 2011;27(12):2441-51.

15. Wen CP, Wu X. Stressing harms of physical inactivity to promote exercise. Lancet. 2012;380(9838):192-3.

16. Barreto S, Passos V, Cardoso A, Lima-Costa M. Quantificando o risco dedoença coronariana na comunidade. Projeto Bambuí. Arq Bras Cardiol. 2003;81(6):549-55.

17. Intituto Brasileiro de Geografia e Estatística. Cidades, 2015 [acesso em 2015 fev 02]. Disponível emhttp:// cidades.ibge.gov.br/xtras/perfil.php?codmun=314610.

18. Matsudo S, Araujo T, Matsudo V, Andrade D, Andrade E, Oliveira LC, et al. Questionario internacional de atividade física (IPAQ): estudo de validade e 20 reprodutibilidade no Brasil. Rev Bras Ativ Fís Saúde. 2001;6(2):5-18

19. Ainsworth BE, Haskell WL, Whitt MC, Irwin ML, Swartz AM, Strath SJ, et al. Compendiumof physical activities: an update of activity codes and MET intensities. Med Sci Sports Exerc. 2000;32(9 Suppl):S498-504

20. Haskell WL, Lee IM, Pate RR, Powell KE, Blair SN, Franklin BA, et al. Physical activity and public health: updated recommendation for adults from the American College of Sports Medicine and the American Heart Association. Med Sci Sports Exerc. 2007;39(8):1423-34.

21. Ministério da Saúde. Pesquisa Nacional de Saúde 2013. Percepção do estado de saúde, estilos de vida e 
doenças crônicas. Brasil, Grandes Regiões e Unidades da Federação. Rio de Janeiro:IBGE; 2014.

22. Ministério da Saúde. Secretaria de Vigilância em Saúde. Secretaria de Gestão Estratégica e Participativa. Vigitel Brasil 2013: vigilância de fatores derisco e proteção para doenças crônicas por inquérito telefônico. Brasília: Ministério da Saúde; 2014.

23. Tassitano RM, Feitosa WMN, Santiago Júnior GL,Tenório MCM.Simultaneidade de comportamentos de risco a saúde e fatores associados emtrabalhadores da indústria. Rev Bras Ativ Fis Saude. 2010;15(1):42-49.

24. Petrović B, Jasarević-Komljenović Z, Srdić B, Stokić E. Cardiovascular risk factors in workers at health centre in Beocin. Med Pregl. 2008;61(5-6):257-62

25. Morris LJ, D'Este C, Sargent-Cox K, Anstey KJ. Concurrent lifestyle riskfactors: Clusters and determinants in an Australian sample. Prev Med. 2016;84:1-5.

26. Lachat C, Otchere S, Roberfroid D, Abdulai A, Seret FM, Milesevic J, et al. Diet and physical activity for the prevention ofnoncommunicable diseases in low- and middle-income countries: a systematic policyreview. PLoS Med. 2013;10(6):e1001465.

27. Pinho CPS, DIniz AS, Arruda IKG, Llra PIC, Cabral PC, Siqueira LAS, et al. Consumo de alimentos protetores e preditores do risco cardiovascular emadultos do estado de Pernambuco. Rev Nutr. 2012;25(3):341-51

28. Kolbe-Alexander TL, Conradie J, Lambert EV. Clustering of risk factors fornon-communicable disease and healthcare expenditure in employees with privatehealth insurance presenting for health risk appraisal: a cross-sectional study. BMC Public Health. 2013;13:1213.

29. Adams PF, Kirzinger WK, Martinez M. Summary health statistics for the U.S. population: National Health Interview Survey, 2012. Vital Health Stat 10. 2013;(259):1-95.

30. Garcia LM, Barros MV, Silva KS, Del Duca GF, Costa FF, Oliveira ES, et al.Socio-demographic factors associated with three sedentary behaviors in Brazilian workers. Cad Saude Publica. 2015;31(5):1015-24. 